\title{
Stand-alone signal transmission based on electromagnetic resonant wireless power transmission system
}

\author{
DENG Yafeng ${ }^{1, a}$, LI Jian², LI Quanlai ${ }^{3}$ \\ ${ }^{1}$ School of Material and Mechanical Engineering, Beijing Technology and Business University, \\ Beijing, China

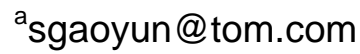

Keywords: electromagnetic resonant; wireless power transmission; signal transmission; energy transmission

\begin{abstract}
Physical connection between devices prone to failure in many applications. In the paper, energy coils and data coils are placed independently on the basis of electromagnetic resonant wireless power transmission technology, At the same time, resonant frequency of energy coils and data coils are designed to be the same, energy is one-way transmission from primary side to secondary side, signal can achieve two-way transmission through data coils. Experimental results show that magnetic coupling between energy coils and data coils impacts energy and data transmission slightly in resonance condition and good simultaneous transmission performance can be gained.
\end{abstract}

\section{Introduction}

Electromagnetic resonance wireless power transmission technology is a new field in both domestic and international academia, It focuses on the basic research and application of electromagnetic field, power electronics, high frequency electron, electromagnetic induction, coupling theory and so on. It has good prospect in mobile phone, notebook computer and electric vehicle charging, it is a new technology with wide application range ${ }^{[1][2][3]}$.At this stage, the research on the electromagnetic resonance wireless power transmission technology is in the initial stage, theoretical and experimental studies are still relatively small. In this paper, the experimental study of the synchronous transmission of the independent energy and signal based on the electromagnetic resonance wireless power transmission system is firstly carried out. And the experimental results are compared with the experimental results of high frequency signal transmission based on electromagnetic induction wireless power transmission system.

\section{Coupling performance analysis}

Resonance is widely found in nature,According to the theory of maximum energy transfer theorem and harmonious vibration, When working frequency is the same as the natural frequency of the system (primary and secondary circuit), the maximum transmission efficiency can be obtained.The basic principle of the electromagnetic resonance wireless power transmission system is to make the frequency of the high frequency power source and the natural frequency of the primary coil, thus constituting a high frequency magnetic coupling resonance system.The electromagnetic field increases rapidly with the increase of the distance. The electromagnetic resonance wireless power transmission is to capture the electromagnetic field which is caused by the electromagnetic field with the range of the distance.

Electromagnetic resonant wireless power transmission system has high frequency power source and load in addition to the initial stage of coil.For the sake of simplicity, the primary coil is directly used as the object of study.As shown in Figure 1, the LC series resonant coupling model is illustrated.The U1 is high frequency power source, $\mathrm{R} \rho 1$ and $\mathrm{R} \rho 2$ are the primary, secondary resonant inductance coil resistance, C1 and C2 are respectively series resonant capacitor, RL is load, L1 and 
L2 are respectively the inductance of the primary and secondary coils, $\mathrm{M}$ is the mutual inductance between the primary and secondary coils, $\mathrm{d}$ is the distance transmission.

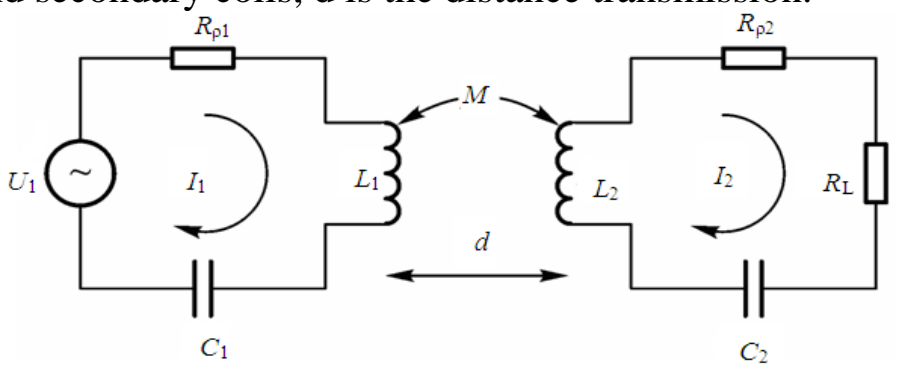

Fig.1 Resonant coupling circuit

Figure 1 gives the positive direction of the current, the secondary circuit equation is:

$$
\left\{\begin{array}{l}
I_{1}\left(\mathrm{j} \omega L_{1}+R_{\rho 1}\right)-I_{2} \mathrm{j} \omega M=U_{1} \\
I_{2}\left(\mathrm{j} \omega L_{2}+R_{\mathrm{\rho} 2}+R_{\mathrm{L}}\right)=I_{1} \mathrm{j} \omega M
\end{array}\right.
$$

It can be seen from formula (1), transmission efficiency of electromagnetic resonant wireless power transmission system is related to the system resonance frequency $\omega$, the primary coil resistance $R_{\rho 1}$, the secondary coil resistance $R_{\rho 2}$, load resistance $R_{\mathrm{L}}$ and mutual inductance $M$.Therefore, it is very important to determine the inductance of the primary and secondary coils in the calculation of the transmission efficiency of the electromagnetic resonant wireless power transmission system.In the design process, in addition to the production process will lead to the deviation of the inductance of the theoretical calculation, the environment of the coil, the parasitic parameters of the circuit and the circuit temperature which rise will lead to changes in the amount of coil inductance.

Another important parameter that affects the transmission efficiency of the electromagnetic resonance type wireless power transmission system is the mutual inductance $M$, and the calculation formula of the mutual inductance of the resonance state is ${ }^{[2]}$ :

$$
M=\frac{\pi}{2} \cdot \frac{\mu_{0} \sqrt{N_{1} N_{2}}\left(r_{1} r_{2}\right)^{2}}{d^{3}}
$$

In the formula:

$$
\mu_{0} \longrightarrow \text { space permeability; }
$$

$N_{1}, N_{2}$ - turns of primary and secondary coils;

$r_{1} 、 r_{2} \longrightarrow$ radius of the primary and secondary coils;

$d$ - distance between primary and secondary coils.

It can be seen from formula (2), the mutual inductance value of the electromagnetic resonance wireless power transmission system is inversely cube proportional to the distance between the primary coils. That is, the greater the distance, the smaller the value of mutual inductance, the more the system coupling performance, the lower the transmission efficiency of the corresponding system.

\section{Independent signal transmission analysis}

The independent type of signal transmission system of the energy coil and data coil is independently, as shown in figure 2. Energy is transmitted from the primary side to the secondary side, and the signal is transmitted through a signal coil. The method of adding a pair of signal transmission coils is outside the energy transmission coil ${ }^{[4]}$ 。 


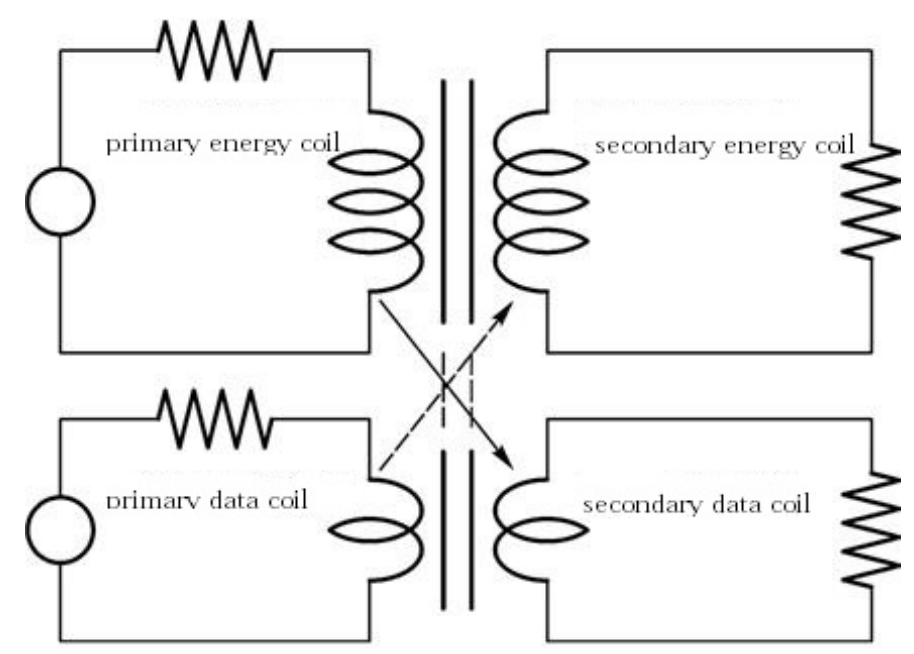

Fig.2 Stand-alone signal transmission schematic diagram

For primary and secondary coils, there are two types of energy coils and data coils which placed in the same way as the primary and secondary coils, and the energy coils and data coils are arranged in a vertical or parallel manner, as shown in Figure 3.
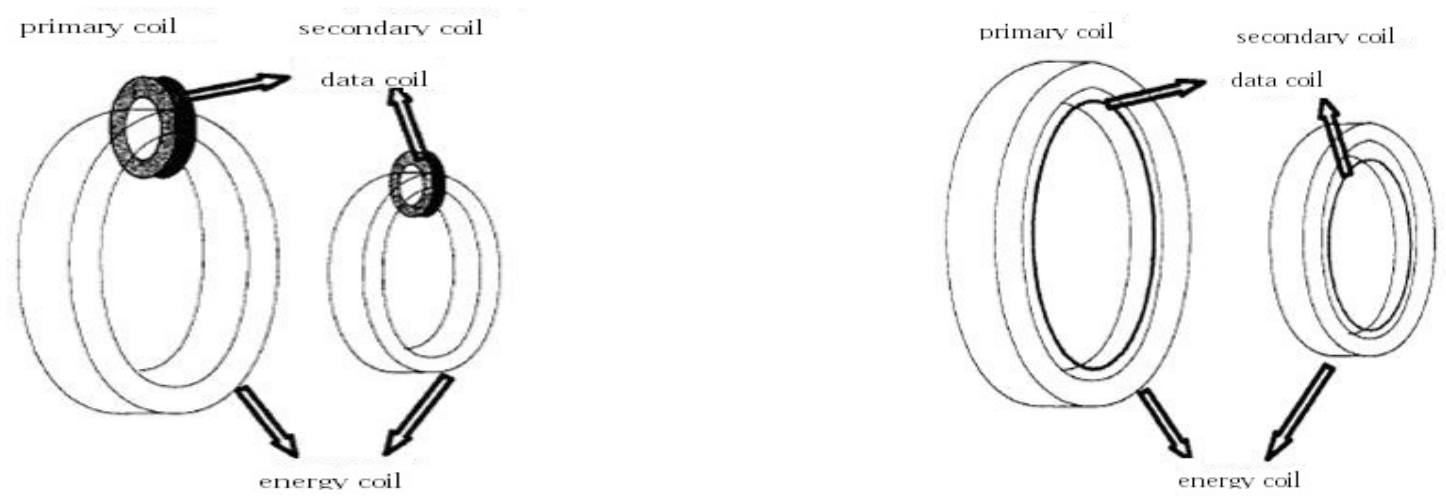

(a)vertical placement of energy coil and data coil (b)parallel placement of energy coil and data coil Fig.3 Placement of Energy coils and data coils

For the vertical manner,the advantage is in the same side, the energy coil and the data is not the ideal state of the coil without coupling, the interaction is not affected.In addition to the coupling between the primary energy coil and the primary data coil, the primary energy coil and the secondary coil, the primary data coil, the primary data coil, and the secondary energy coil are also coupled.

For the case that the energy coil and the data coil are parallel to each other, the advantage is that the structure of the coil is smooth and easy to install. The disadvantage is that: for the first, the secondary energy coil and the data coil, any one coil will be coupled with the other three coils, the energy will be emitted by the coil coupling on the data coil, causing a lot of energy loss, and serious interference with the data signal transmission and reception.

\section{Experimental verification}

Table 1 is a parameter for the data coil and the energy coil, and the experiment is used in parallel with the coil and the energy coil. In the experiment, the voltage of the energy signal is $10 \mathrm{~V}$, the signal voltage is $5 \mathrm{~V}$, the transmission distance is $10 \mathrm{~cm}$. 
Tab.1 Parameters of Energy coils and data coil

\begin{tabular}{c|c|c|c|c|c|c}
\hline & $\begin{array}{c}\text { radiusr } \\
/ \mathrm{mm}\end{array}$ & $\begin{array}{c}\text { turn } / N \\
\text { radius of } \\
\text { wire section } \\
a / \mathrm{mm}\end{array}$ & $\begin{array}{c}\text { theoretical } \\
\text { inductance } \\
/ \mu \mathrm{H}\end{array}$ & $\begin{array}{c}\text { resonant } \\
\text { frequency } \\
f / \mathrm{MHz}\end{array}$ & $\begin{array}{c}\text { matching } \\
\text { capacitor } \\
\mathrm{C} / \mathrm{NF}\end{array}$ \\
\hline $\begin{array}{c}\text { data } \\
\text { coils }\end{array}$ & $\begin{array}{c}r_{1}=r_{2}= \\
65\end{array}$ & 4 & 1 & 3.65 & 0.667 & 9.4 \\
\hline $\begin{array}{c}\text { energy } \\
\text { coils }\end{array}$ & $\begin{array}{c}r_{1}=r_{2}= \\
115\end{array}$ & 4 & 1 & 8.49 & 1.17 & 2.2 \\
\hline
\end{tabular}

In Figure 4, it is the comparison of the waveforms received by the secondary energy coils before and after the addition of the data.In the experiment, the frequency of the energy signal and data signal is set to the resonance frequency of the system.After the system is added to the data waveform, the waveform of the secondary energy coil is little change, and the waveform distortion is not serious, the secondary energy waveform is not affected by the data signal, and The magnetic circuit of the energy coil and the data coil is not obvious to the energy signal.

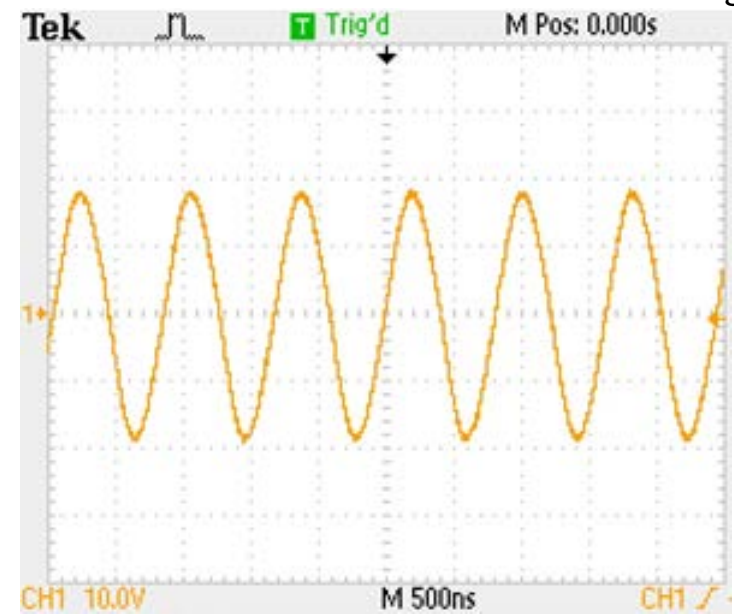

(a) before entering data signal

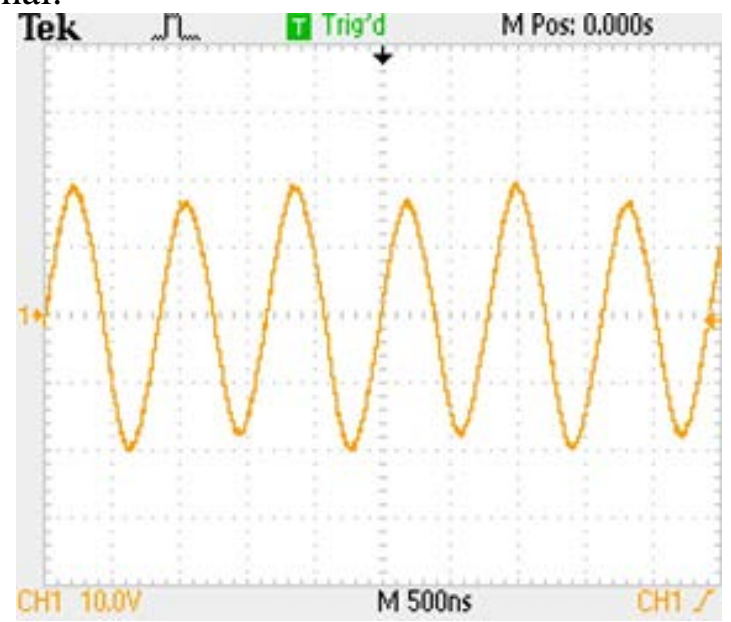

(b) after entering data signal

Fig. 4 Comparison of energy signal waveform when data coils are added

In Figure 5,the waveform of the frequency of the next stage of the data coil received by the different frequency of the time when the energy signal and the data signal are transmitted at the same time, The frequency of data signal is close to the resonance frequency of the signal coil, the signal waveform is better, and the waveform distortion is smaller. So the experimental results show that the coupling magnetic circuit of the energy and data coils can work at the resonance frequency, and the coupled magnetic circuit formed by the energy coil and the data coil is less affected by the transmission of energy and data,it can get very good synchronous transmission effect.

Compared with the literature [5],The experimental results of the independent signal transmission system based on electromagnetic resonance wireless power transmission system are better, and the waveform distortion is not very serious.

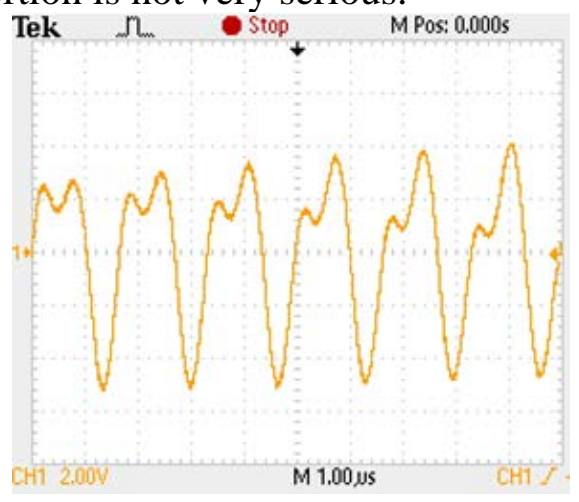

(a) $f=600 \mathrm{kHz}$

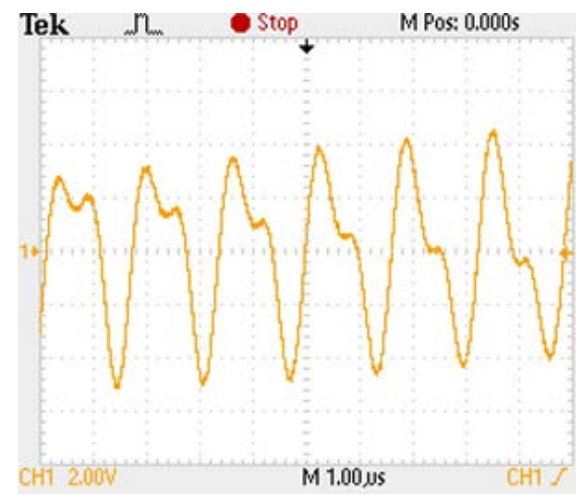

(b) $f=625 \mathrm{kHz}$ 


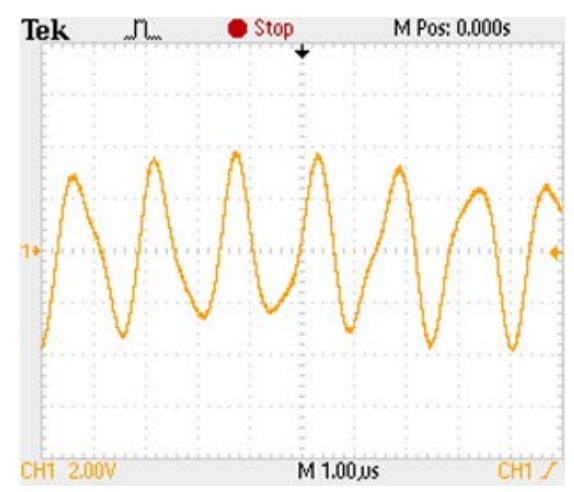

(c) $f=660 \mathrm{kHz}$
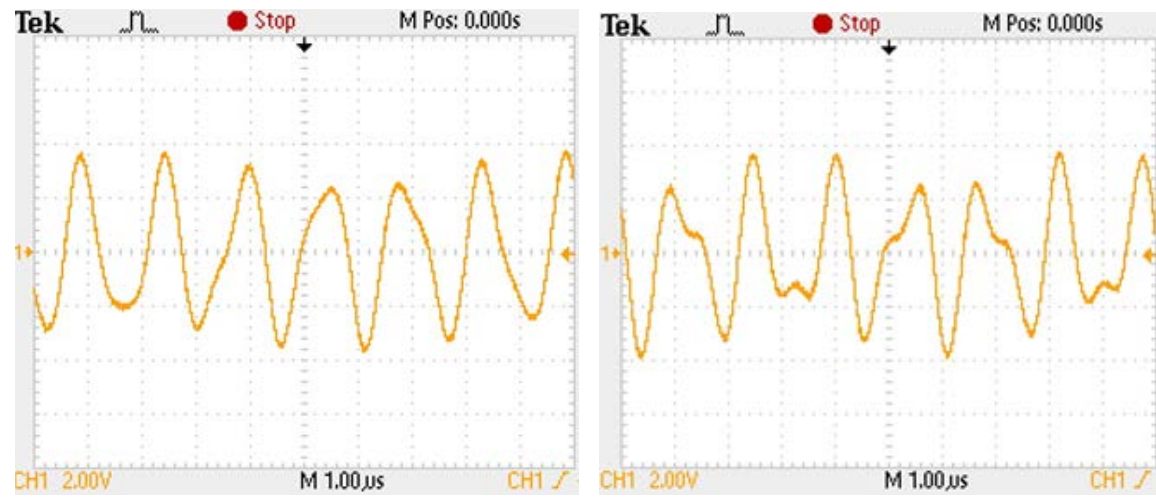

(d) $f=670 \mathrm{kHz}$

(e) $f=700 \mathrm{kHz}$

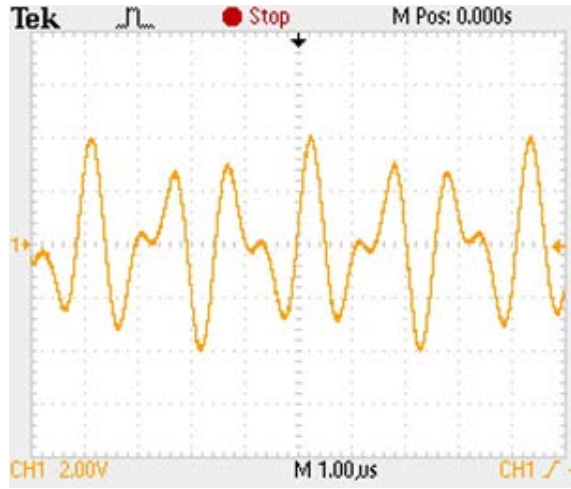

(f) $f=725 \mathrm{kHz}$

Fig.5 Waveform which secondary coil received when frequency are different

\section{Conclusion}

In this paper, an independent signal transmission based on the electromagnetic resonance type wireless power transmission system is firstly studied.Through experiments, in the case of the energy coil and the data coil, the magnetic circuit of the energy and the data is less than the energy and data transmission.Compared with high frequency injection signal transmission experiment, the effect of the independent signal transmission experiment based on the electromagnetic resonance wireless power transmission system is better, and the waveform coefficient is better.

\section{Acknowledgment}

This work is supported by Beijing Municipal Commission Education Scientific and Technological Innovation Project(No.KM201510011005,No.KM201310011003). 


\section{Reference}

[1] Litao Ren.Research On the Power Characteristic of Magnetic Resonance Based Wireless Power Transfer. Harbin: Harbin Institute of Technology[D], 2009.

[2] Karalis A, Joannopoulos J D, Soljaeia M.Efficient wireless non-radiative mid-range energy transfer[J].Annals of Physics, 2008, 3(23):34-38.

[3] Soljaeia M, Rafif E H, Karalis A.Coupled-mode theory for general free-space resonant scattering of waves[J].Physcial Review 2007, 75(5):1-5.

[4] Kuo-kai Shyu, Ko-Wen Jwo, Zhang, Zheng-Yong Chen, et a.l Inductive Power Supply System with Fast Full-Duplex Information Rate Device[J]. Computer as aToo, 12007 (9): 1382-1386.

[5] DENG Yafeng, LI Feng, ZHANG Xupeng, SHI Pengfei.Signal transmission based on electromagnetic inductive wireless power supply system. Machinery Design \& Manufacture:2010(11):34-36. 